\title{
Distributed Turbo Coding in the Presence of Inter-User Channel Impairment
}

\author{
Mohammed El-Hajjar, Osamah Alamri and Lajos Hanzo \\ School of Electronics and Computer Science, University of Southampton, SO17 1BJ, UK. \\ Email: $\{1 \mathrm{~h}\} @ e c s . s o t o n . a c . u k$, \\ http: //www-mobile.ecs.soton.ac.uk
}

\begin{abstract}
We propose a Distributed Turbo Coding (DTC) scheme, where two users cooperate in a two-phase cooperation scenario constituted by the exchange of data between the users in the first phase followed by the uplink transmission of the two users' data in the second phase of cooperation. Following the information exchange phase, the data transmitted from the second user's transmitter during the second phase is an interleaved version of the first user's data. Each user's transmitter is equipped with a Recursive Systematic Convolutional (RSC) code, an interleaver and a Sphere Packing (SP) mapper. At the receiver side, interference cancellation is employed, which outputs two branches of decoded data corresponding to the data transmitted from the two users' transmitters. Iterative detection is employed by exchanging extrinsic information between the RSC decoder and the SP demapper in each decoding branch, as well as between the RSC decoders in the two branches. The proposed DTC scheme is benchmarked against another cooperative scheme, where the two users do not exchange their data, they rather transmit their data simultaneously. At the receiver of the benchmark scheme, iterative detection is carried out by exchanging extrinsic information between the SP demapper and the RSC decoder of each user, but no iterations are invoked between the RSC decoders in the two branches. The proposed scheme attains an $E_{b} / N_{0}$ gain of about $25 \mathrm{~dB}$ at BER of $10^{-5}$, when compared to the benchmark scheme, while considering a perfect Inter-User Channel (IUC) and employing $I_{s y s}=5$ system iterations in conjunction with an interleaver depth of $D_{i n t}=80,000$ bits. Additionally, we study the effects of both Gaussian as well as Ricean IUC on the achievable BER performance of the uplink DTC scheme.
\end{abstract}

\section{INTRODUCTION}

Wireless channels suffer from multipath propagation of signals, that results in a variation in the received signal strength, when the transmitter or receiver are in motion [1]. During severe fading of a specific propagation path, the received signal cannot be correctly decoded, unless some less attenuated multipath versions of it are available at the decoder side. This can be arranged by introducing transmit diversity [2]. In conventional Multiple-Input Multiple-Output (MIMO) systems $[3,4]$ constituted by colocated MIMO elements, transmit diversity is achieved by transmitting different versions of the signal from different antennas located at the same transmitter or receiver station. The antenna spacing in colocated MIMOs is assumed to be sufficiently large so that the assumption of statistical independence of the different paths from the different antennas is justified. However, satisfying the assumption of a sufficiently high antenna spacing may be impractical for

The financial support of Vodafone under the auspices of the Dorothy Hodgkin Postgraduate Award and that of the of the European Community under Seventh Framework Programme grant agreement ICT OPTIMIX nINFSOICT-214625 is gratefully acknowledged. shirt-pocket-sized wireless devices, which are typically limited in size and hardware complexity to a single transmit antenna.

Hence, we can surmise that transmit diversity methods are not readily applicable to compact wireless communicators owing to the size as well as complexity constraints that limit the use of multiple transmit antennas. Recently, cooperative communication techniques [5-7] were proposed for eliminating correlation amongst the diversity paths by cooperatively activating the single antenna of several Mobile Stations (MS), hence effectively creating a distributed MIMO scheme. In other words, single-antenna aided users support each other by "sharing their antennas" [8] and thus generate a virtual multiantenna environment [9]. In cooperative communications it is possible to guarantee that the cooperating users are sufficiently far apart, in order to attain independent fading. Since the signals transmitted from different users undergo independent fading, spatial diversity can be achieved by the concerted action of the cooperating partners' antennas.

Additionally, it was proposed in [10] to use distributed space-time codes for the relay channel, demonstrating its benefits from an information theoretic point of view. In [11] Janani et al. proposed space-time cooperation in addition to implementing turbo coding by exchanging extrinsic information between the data received from the source and the relay. Furthermore, a method designed for achieving cooperative diversity using rate compatible punctured codes was proposed in $[12,13]$. In $[14,15]$, it was proposed to employ distributed turbo codes by exchanging extrinsic information between the data received from the source and that received from the relay, where the relay applies interleaving for the data received from the source and then uses an appropriate channel code before retransmission.

In this paper we design a Distributed Turbo Coding (DTC) scheme, where two users cooperate in a two-phase cooperation scenario. During the first phase of cooperation, each user sends his/her own data to the other user, followed by the second phase, where both users transmit their own data as well as the data of the other user after interleaving and channel coding. The two users employ multidimensional Sphere Packing (SP) modulation [16] and then transmit their data simultaneously. We employ SP modulation for the sake of attaining further iteration gains, as described in [16]. The data transmitted from the second user's transmitter is an interleaved version of the bit stream transmitted from the first user's transmitter. The receiver applies interference cancellation for the sake of eliminating the interference imposed by one user's data on the other user's data. Hence, at the receiver side, the 


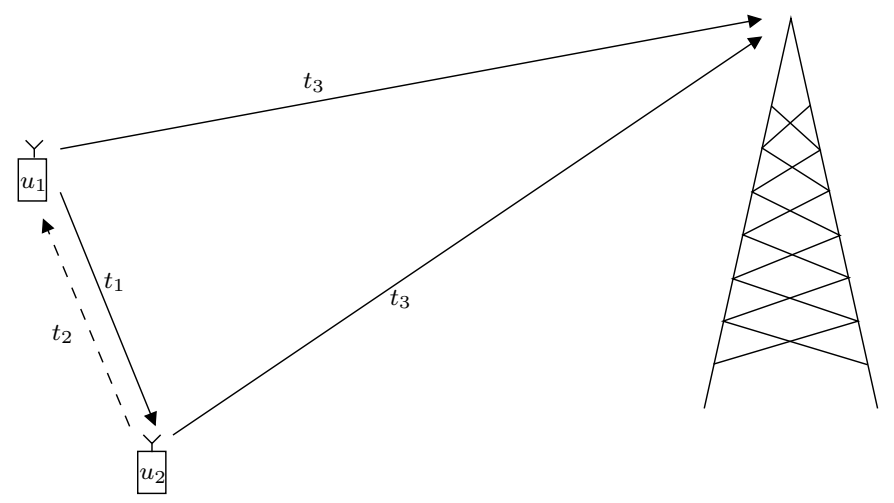

Fig. 1. Distributed turbo coding system model.

interference canceller outputs two data streams corresponding to the data transmitted from the first and the second users' transmitters, respectively. Afterwards, iterative detection is employed by exchanging extrinsic information between the SP demapper and the channel decoder in each data stream (or decoding branch) as well as between the two channel decoders of the two branches, hence forming a distributed turbo code. Additionally, we study the effects of different InterUser Channel (IUC) characteristics on the attainable BER performance of the proposed DTC system, when the IUC is perfect, Gaussian and Ricean faded.

The rest of this paper is organised as follows. In Section II we describe the proposed DTC system followed by Section III, where we characterise the DTC aided system with the aid of simulation results, while considering different IUC characteristics. Finally, we present our conclusions in Section IV.

\section{Distributed TURbo CODING}

The proposed DTC scheme is based on the system architecture shown in Figure 1, where the users $u_{1}$ and $u_{2}$ cooperate in a two-phase cooperation scenario. The difference between our proposed system and those presented in [11-15] is that the two users in our design transmit their own data in addition to the other user's data, while the systems in [11-15] have a single active user and a relay transmitting the data of the active user only. Additionally, in our proposed system we study the effect of the IUC characteristics on the attainable performance of the proposed DTC system, while the schemes of [11-15] assume perfect IUC. As shown in Figure 1, two users cooperate in order to communicate with a Base Station (BS). In the proposed scheme, the two users cooperate in two phases, where they exchange their data in the first cooperation phase and then they both transmit simultaneously their own data as well as the data of the other user in the second phase of cooperation.

The proposed system operates in a realistic half-duplex mode, where none of the transceivers can transmit and receive at the same time. The transceivers operate in a Time Division Duplex (TDD) mode, where different transmitters transmit in different time slots. As shown in Figure 1, in time slot $t_{1}$ user 1 transmits his/her data to user 2. Similarly, in time slot $t_{2}$ user 2 shares his/her data with user 1 . Hence, the first two

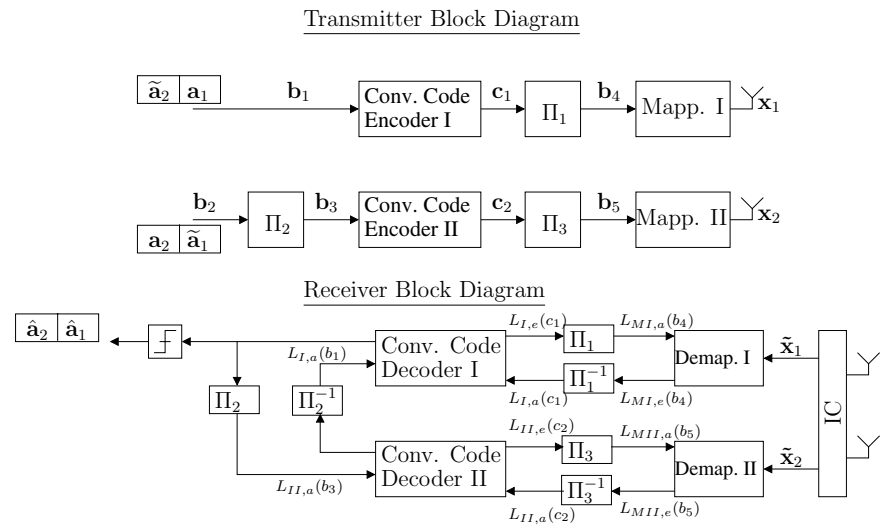

Fig. 2. Block diagram of the two users' transmitters and the BS receiver.

time slots, i.e. time slots $t_{1}$ and $t_{2}$, comprise the first phase of cooperation. In the second phase of cooperation, i.e. in time slot $t_{3}$, the two users transmit their data simultaneously to the BS after interleaving and channel coding.

A more detailed block diagram of the proposed scheme is shown in Figure 2. In time slot $t_{1}$, user 1 transmits his/her bit stream $\mathbf{a}_{1}$ to user 2 , where the received and decoded estimate of $\mathbf{a}_{1}$ is denoted by $\widetilde{\mathbf{a}}_{1}$. Hence, the Decode-andForward signalling strategy $[5,6]$ is used in the proposed DTC scheme. Similarly, in time slot $t_{2}$ user 2 transmits his/her data bit stream $\mathbf{a}_{2}$ to user 1 , where the received and decoded bit stream in denoted by $\widetilde{\mathbf{a}}_{2}$.

Once the inter-user communication phase was concluded during time slots $t_{1}$ and $t_{2}$, the two users now have both their own data as well as an estimate of the data of the other user. Both users concatenate their own data with the estimate of the other user's data, as shown in Figure 2 and detailed as follows. The first user appends the estimate of the second user's data $\widetilde{\mathbf{a}}_{2}$ with his/her own data $\mathbf{a}_{1}$ and then encodes the resultant bit stream $\mathbf{b}_{1}$ by a Recursive Systematic Convolutional (RSC) code. The channel coded bit stream $\mathbf{c}_{1}$ is interleaved by the random bit interleaver $\Pi_{1}$ of Figure 2 and then the interleaved bits $\mathbf{b}_{4}$ are modulated by the SP Mapper I of Figure 2. Similarly, user 2 appends his/her own data $\mathbf{a}_{2}$ to the estimate of user 1's data $\widetilde{\mathbf{a}}_{1}$ and then interleaves the resultant bit stream $\mathbf{b}_{2}$ by the bit interleaver $\Pi_{2}$ of Figure 2 . The interleaved bit stream $\mathbf{b}_{3}$ is channel coded by a RSC code and then the encoded bit stream $\mathbf{c}_{2}$ is interleaved. Finally, the interleaved bit stream $\mathbf{b}_{5}$ is modulated by the SP Mapper II of Figure 2.

The two users simultaneously transmit their SP modulated symbol streams $\mathbf{x}_{1}$ and $\mathbf{x}_{2}$, assuming that there is perfect synchronisation between the two users' MSs. The two users' MSs normalise their transmit power so that the total transmit power in the two phases of cooperation remains equivalent to the case, when there is no cooperation.

At the BS, low-complexity Zero Forcing (ZF) Interference Cancellation (IC) is applied, as described in [4]. The IC decoder outputs the two streams of decoded data $\widetilde{\mathbf{x}}_{1}$ and $\widetilde{\mathbf{x}}_{2}$ corresponding to the data transmitted by user 1 and user 2 , respectively, as shown in Figure 2. After the IC stage, estimates 


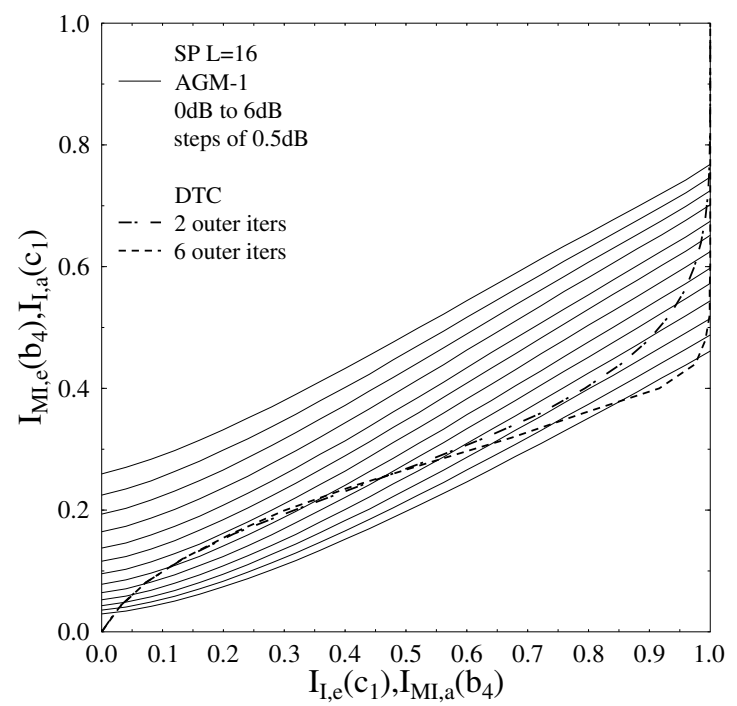

Fig. 3. EXIT chart of the DTC system, where cooperation is employed between two users employing 1/2-rate RSC codes while uplink communication is performed over a narrowband Rayleigh fading channel. The EXIT curves were generated for the perfect IUC case.

of the transmitted data streams $\widetilde{\mathbf{x}}_{1}$ and $\widetilde{\mathbf{x}}_{2}$ are passed to the SP demappers of Figure 2. The SP demappers I and II of Figure 2 utilise the data received from the IC together with the a priori information $L_{M I, a}\left(\mathbf{b}_{4}\right)$ and $L_{M I I, a}\left(\mathbf{b}_{5}\right)$ passed to them from the RSC decoders for the sake of providing the improved extrinsic information $L_{M I, e}\left(\mathbf{b}_{4}\right)$ and $L_{M I I, e}\left(\mathbf{b}_{5}\right)$, which is then passed to the RSC decoders as the a priori information $L_{I, a}\left(\mathbf{c}_{1}\right)$ and $L_{I I, a}\left(\mathbf{c}_{2}\right)$ after deinterleaving. Afterwards, iterative detection is carried out by exchanging extrinsic information between the two RSC decoders of the two branches. The two RSC decoders employ iterative detection for the sake of providing improved extrinsic information to the SP demappers of Figure 2. The iterations employed between the two RSC decoders are similar in terms of their concept to that employed in classic turbo codes $[2,17]$, which motivates the terminology of distributed turbo coding.

Figure 3 shows the EXIT chart of the proposed DTC scheme of Figure 2. In Figure 3 we plot the EXIT curve of the inner SP demappers in conjunction with $L=16$ and the AntiGray Mapping AGM-1 defined in [18]. The EXIT curves of the inner SP demappers seen in Figure 3 are shown for $E_{b} / N_{0}$ values between $0 \mathrm{~dB}$ and $6 \mathrm{~dB}$ in steps of $0.5 \mathrm{~dB}$. Figure 3 also shows the inverted EXIT curve of the outer DTC. The outer codes applied are 1/2-rate memory-2 RSC codes in conjunction with an octal generator polynomial of $\left(G_{r}, G\right)=(7,5)_{8}$, where $G$ is the feedforward polynomial and $G_{r}$ is the feedback polynomial. We plot two curves corresponding to the outer DTC in Figure 3, where the one represented by the dashed-dotted line evolving mostly above the dashed curve corresponds to two iterations between the two outer RSC decoders, while the other curve marked by the dashed line corresponds to six iterations between the two RSC codes' decoders. In this case, in the first phase of cooperation, each user modulates the source bits and transmits the QPSK modulated symbols to the other user without incorporating any

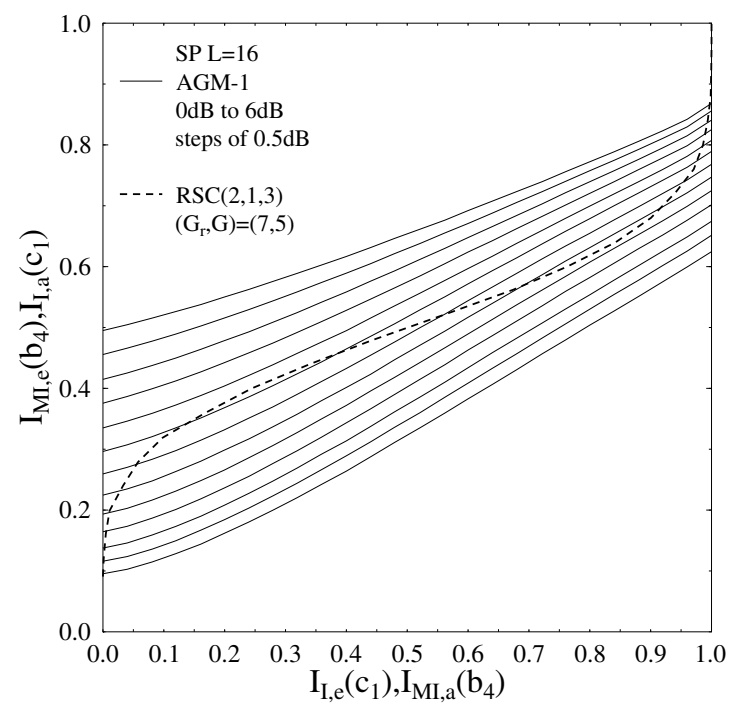

Fig. 4. EXIT chart of the benchmark scheme where iterative detection is carried out between the outer RSC decoder and the inner SP demapper of each user without employing any iteration between the RSC decoders of the two users. In this case, each user transmits his/her own data only and hence there is no inter-user communication involved.

channel coding. Additionally, the EXIT curves are plotted for the system where the IUC is considered to be perfect. As shown in Figure 3, an open convergence tunnel is formed around $E_{b} / N_{0}=3.0 \mathrm{~dB}$. This implies that according to the predictions of the EXIT chart seen in Figure 3, the iterative decoding process is expected to converge for $E_{b} / N_{0}>3.0 \mathrm{~dB}$. On the other hand, observe in Figure 3 that the point of intersection between the EXIT curves of the inner and the outer codes approaches the 1.0 mutual information points of the $x$-axis, as the number of outer iterations or distributed turbo coding iterations increases from $I_{\text {out }}=2$ to 6 . Hence, it is expected that employing $I_{\text {out }}=6$ outer iterations between the RSC decoders will result in a better BER performance than $I_{\text {out }}=2$.

On the other hand, Figure 4 shows the EXIT curve of the benchmark scheme, where two users cooperate by transmitting their own data simultaneously without transmitting the other user's data. In other words, each user encodes his/her own bit stream by an outer RSC code and then the encoded bit stream is interleaved by a random bit interleaver. Afterwards, the interleaved bit stream is modulated by a SP mapper and then transmitted from the users' single-antenna-aided MS. At the receiver side, IC is employed in order to eliminate the interference imposed by each of the users' data on the other user's data. Then iterative detection is carried out by exchanging extrinsic information between the outer RSC decoder and the inner SP demapper in each branch and no iterations are employed between the two outer RSC decoders, since the data in the two branches of Figure 2 in this case are different. Figure 4 shows the EXIT chart of the benchmark scheme in conjunction with an inner SP demapper employing $L=16$ and AGM-1 defined in [18] for $E_{b} / N_{0}$ between $0 \mathrm{~dB}$ and $6 \mathrm{~dB}$ in steps of $0.5 \mathrm{~dB}$. The outer code in this case is a $1 / 2$-rate memory-2 RSC code in conjunction with an octal generator polynomial of $\left(G_{r}, G\right)=(7,5)_{8}$. As shown in Figure 4, an 


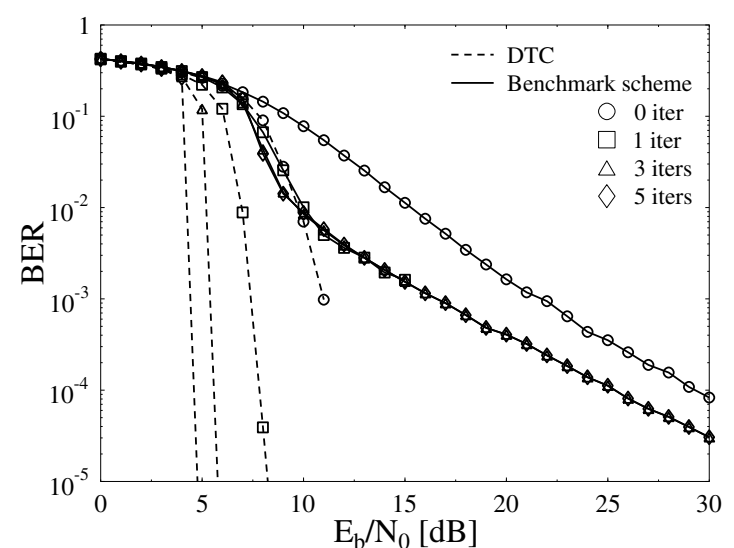

Fig. 5. BER performance comparison of the proposed distributed turbo coded system and the benchmark scheme in conjunction with an interleaver depth of $D_{\text {int }}=80,000$ bits for a variable number of system iterations $I_{s y s}$, where two users are cooperating and communication over an narrowband uplink Rayleigh fading channel. The IUC in this case is considered to be perfect.

open convergence tunnel is formed around $E_{b} / N_{0}=4.0 \mathrm{~dB}$. This implies that according to the predictions of the EXIT chart seen in Figure 4, the iterative decoding process is expected to converge at $E_{b} / N_{0}>4.0 \mathrm{~dB}$. Additionally, a comparison between Figure 3 and Figure 4 shows that the benchmark scheme exhibits a higher error floor than that of the proposed DTC scheme.

\section{RESUlTS AND Discussion}

In this section, we consider the achievable performance of the proposed DTC arrangement, where two users cooperate in order to simultaneously transmit their data and then apply the $\mathrm{ZF}$ IC at the receiver for the sake of eliminating the interference imposed by each of the users' data on the other user's data. Each user's MS is equipped with a single antenna and each applies a 1/2-rate memory-2 RSC code for encoding the bits, which are then mapped to symbols using a SP mapper in conjunction with $L=16$ and the Anti-Gray Mapping AGM-1 defined in [18]. In this section, we consider uplink transmissions over a narrowband uncorrelated Rayleigh fading channel, where coherent detection is applied at the receiver side. It is also assumed that the Base Station's (BS) receiver has perfect knowledge of the uplink channel impulse response.

Figure 5 compares the attainable BER performance of both the proposed DTC scheme and of the benchmark scheme, while considering uplink transmissions over a narrowband Rayleigh fading channel. In this case, the IUC is considered to be perfect, i.e. the data exchanged between the two users is perfectly recovered without any errors. In Figure 5 an interleaver depth of $D_{\text {int }}=80,000$ bits was employed and SP modulation in conjunction with $L=16$ and the AGM-1 of [18] was used. Observe in Figure 5 that the benchmark scheme suffers from a fairly pronounced error floor and that no iteration gain may be obtained after employing more than one inner iteration between the SP demapper and the RSC decoder in each branch. This result confirms the EXIT chart predictions of Figure 4, where the EXIT curves of the inner and outer codes intersect for $E_{b} / N_{0}=6.0 \mathrm{~dB}$ at a point different

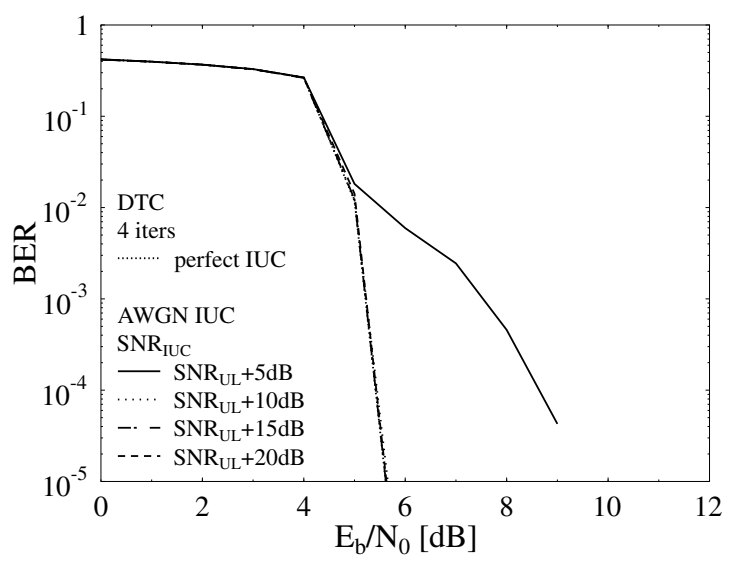

Fig. 6. Comparison of the attainable BER performance of the proposed distributed turbo coding scheme, while considering a AWGN IUC for variable IUC SNR values. The figure corresponds to $I_{s y s}=4$ iterations in conjunction with an interleaver depth of $D_{\text {int }}=80,000$ bits.

from the 1.0 point on the $x$-axis, explicitly indicating the lack of convergence to an infinitesimally low BER. On the other hand, observe in Figure 5 that the proposed DTC aided system exhibits no error floor. This can be explained from the EXIT chart of Figure 3, where the EXIT curves of the inner and outer codes intersect at the 1.0 point on the $x$-axis, when there is an open tunnel. The 1.0 point on the $x$-axis corresponds to the case, where the outer decoder has perfect extrinsic information available at its output. For the proposed DTC scheme, a system iteration $I_{s y s}$ is constituted by a single inner iteration between the SP demapper and the RSC decoder of each branch, followed by $I_{\text {outer }}=6$ iterations between the two RSC decoders in the two branches. Explicitly, the proposed DTC system outperforms its benchmark scheme by $E_{b} / N_{0}$ of about $25 \mathrm{~dB}$ at a BER of $10^{-5}$ after employing $I_{\text {sys }}=5$ system iterations.

Figure 6 shows the attainable BER performance of the proposed DTC scheme, while considering uplink transmission over a narrowband Rayleigh fading channel, where the IUC considered is a Line-Of-Sight (LOS) AWGN channel, i.e. Gaussian noise is added to the received data without any amplitude or phase attenuation. In Figure 6 an interleaver depth of $D_{\text {int }}=80,000$ bits was employed and SP modulation in conjunction with $L=16$ and AGM-1 of [18] was used. Figure 6 also shows the BER performance of the DTC system, when a perfect IUC is considered. The BER curves seen in Figure 6 correspond to the system, where $I_{\text {sys }}=4$ system iterations are employed. The notation $\left(\mathrm{SNR}_{U L}+10 \mathrm{~dB}\right)$ in Figure 6 means that the IUC SNR SNR $I U C$ is $10 \mathrm{~dB}$ higher than the uplink channel SNR, where $\mathrm{SNR}_{U L}$ represents the uplink channel SNR. Observe in Figure 6 that the attainable BER performance of the DTC system, when the IUC is constituted by an AWGN channel having a $10 \mathrm{~dB}$ higher SNR than that of the uplink channel, is equivalent to that of the system considering a perfect IUC. However, when the $\mathrm{SNR}_{I U C}$ is $5 \mathrm{~dB}$ higher than $\mathrm{SNR}_{U L}$, an error floor is formed in Figure 6. This is due to the fact that the iterative detection at the receiver side assumes that the data in the two RSC decoders is identical, while the inter-user communication induces errors 


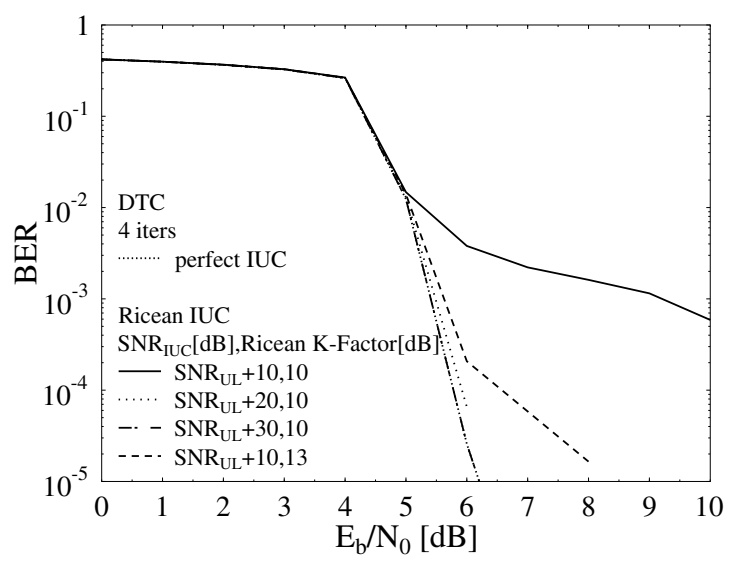

Fig. 7. Comparison of the attainable BER performance of the proposed distributed turbo coding scheme, while considering a Ricean IUC for a variable IUC SNR having different Ricean K-factor. The figure corresponds to $I_{\text {sys }}=4$ iterations in conjunction with an interleaver depth of $D_{\text {int }}=80,000$ bits.

in the data available at the users' terminals and hence results in a discrepancy in the data transmitted from the two users' terminals, which results in the error floor of Figure 6.

Figure 7 shows the attainable BER performance of the proposed DTC scheme, while considering uplink transmissions over a narrowband Rayleigh fading channel, when the IUC considered is a Ricean channel. In Figure 7 an interleaver depth of $D_{\text {int }}=80,000$ bits was employed and SP modulation in conjunction with $L=16$ and AGM-1 of [18] was used. Figure 7 also shows the BER performance of the DTC aided system, when a perfect IUC is considered. The BER curves of Figure 7 correspond to the system where $I_{\text {sys }}=4$ system iterations are employed. Observe in Figure 7 that the attainable BER performance of the DTC assisted system is equivalent to that of the system considering a perfect IUC, when the Ricean $\mathrm{K}$-factor is $10 \mathrm{~dB}$ and $\mathrm{SNR}_{I U C}$ is $30 \mathrm{~dB}$ higher than the uplink channel SNR. However, when the IUC SNR becomes less than $20 \mathrm{~dB}$ higher than the uplink channel SNR, the BER performance degrades and an error floor is formed. As the Ricean K-factor increases to $13 \mathrm{~dB}$, no error floor is formed in the BER curve even for $\mathrm{SNR}_{I U C}$ which is only $10 \mathrm{~dB}$, rather than $20 \mathrm{~dB}$ higher than the uplink channel SNR.

\section{CONCLUSION}

In this paper we proposed a distributed turbo coding scheme, where each user's single-antenna aided transmitter is constituted by a RSC code, an interleaver and a SP mapper. The two users cooperate in two phases, where the first phase of cooperation is constituted by the exchange of data between the two users in two time slots followed by the simultaneous transmission of data from the two users' MSs. At the receiver side, iterative detection is carried out between the SP demapper and the RSC decoder as well as between the two RSC decoders of the two users. The proposed DTC scheme is benchmarked against another cooperative scheme, where the two users do not exchange their data, they rather transmit their data simultaneously. At the receiver of the benchmark scheme, iterative detection is carried out between the SP demapper and the RSC decoder of each user. The proposed scheme attained an $E_{b} / N_{0}$ gain of about $25 \mathrm{~dB}$ at BER of $10^{-5}$, when compared to the benchmark scheme, while considering a perfect IUC and employing $I_{\text {sys }}=5$ system iterations in conjunction with an interleaver depth of $D_{\text {int }}=80,000$ bits. Additionally, a study of the inter-user channel characteristics effects on the performance of the proposed uplink DTC scheme was conducted. Explicitly, we studied the effect of considering Gaussian as well as Ricean faded inter-user channels on the attainable performance of the proposed uplink DTC scheme.

\section{REFERENCES}

[1] L. Hanzo, S.X. Ng, T. Keller and W. Webb, Quadrature amplitude modulation: From basics to adaptive trellis-coded, turbo equalised and space-time coded OFDM, CDMA and MC-CDMA systems, 2nd Edition. Chichester, England: John Wiley and Sons Ltd and IEEE Press, 2004.

[2] L. Hanzo, T.H. Liew and B.L. Yeap, Turbo coding, turbo equalisation and space time coding for transmission over fading channels. Chichester, UK: Wiley: IEEE Press, 2002.

[3] V. Tarokh, H. Jafarkhani and A.R. Calderbank, "Space-time block codes from orthogonal designs," IEEE Transactions on Information Theory, vol. 45, no. 5, pp. 1456-1467, 1999.

[4] P.W. Wolniansky, G.J. Foschini, G.D. Golden and R.A. Valenzuela, "VBLAST: an architecture for realizing very high data rates over the richscattering wireless channel," in International Symposium on Signals, Systems and Electronics, (Pisa), pp. 295-300, September 1998.

[5] A. Sendonaris, E. Erkip and B. Aazhang, "User cooperation diversity Part I: System description," IEEE Transactions on Communications, vol. 51, no. 11, pp. 1927-1938, 2003.

[6] A. Sendonaris, E. Erkip and B. Aazhang, "User cooperation diversity Part II: Implementation aspects and performance analysis," IEEE Transactions on Communications, vol. 51, no. 11, pp. 1939-1948, 2003.

[7] G. Scutari and S. Barbarossa, "Distributed space-time coding for regenerative relay networks," IEEE Transactions on Wireless Communications, vol. 4, pp. 2387-2399, September 2005.

[8] A. Nosratinia, T.E. Hunter and A. Hedayat, "Cooperative communication in wireless networks," IEEE Communications Magazine, vol. 42, no. 10, pp. 74-80, 2004.

[9] M. Dohler, E. Lefranc and H. Aghvami, "Space-time block codes for virtual antenna arrays," in The 13th IEEE International Symposium on Personal, Indoor and Mobile Radio Communications, vol. 1, pp. 414417, September 2002.

[10] J.N. Laneman and G.W. Wornell, "Distributed space-time-coded protocols for exploiting cooperative diversity in wireless networks," IEEE Transactions on Information Theory, vol. 49, no. 10, pp. 2415-2425, 2003.

[11] M. Janani, A. Hedayat, T. Hunter and A. Nosratinia, "Coded cooperation in wireless communications: space-time transmission and iterative decoding," IEEE Transactions on Signal Processing, vol. 52, pp. 362-371, February 2004.

[12] T.E. Hunter and A. Nosratinia, "Diversity through coded cooperation," IEEE Transactions on Wireless Communications, vol. 5, pp. 283-289, February 2006.

[13] T.E. Hunter and A. Nosratinia, "Cooperation diversity through coding," in IEEE International Symposium on Information Theory, 2002.

[14] M.C. Valenti and B. Zhao, "Distributed turbo codes: towards the capacity of the relay channel," in IEEE Vehicular Technology Conference VTCFall, vol. 1, pp. 322-326, October 2003.

[15] B. Zhao and M.C. Valenti, "Distributed turbo coded diversity for relay channel," Electronics Letters, vol. 39, pp. 786-787, May 2003.

[16] O. Alamri, B.L. Yeap and L. Hanzo, "A turbo detection and spherepacking-modulation-aided space-time coding scheme," IEEE Transactions on Vehicular Technology, vol. 56, pp. 575-582, March 2007.

[17] C. Berrou, A. Glavieux, "Near optimum error correcting coding and decoding: turbo-codes," IEEE Transactions on Communications, vol. 44, pp. 1261-1271, October 1996

[18] M. El-Hajjar, O. Alamri, S. X. Ng and L. Hanzo, "Turbo detection of precoded sphere packing modulation using four transmit antennas for differential space-time spreading," IEEE Transactions on Wireless Communications, vol. 7, pp. 943-952, March 2008. 